\title{
Global Existence and Blowup for a Reaction-Diffusion System with Nonlocal Boundary Condition
}

\author{
Jun Zhou \\ School of Mathematics and Statistics, Southwest University, Chongqing 400715, China \\ Correspondence should be addressed to Jun Zhou; jzhouwm@gmail.com
}

Received 23 April 2013; Accepted 16 May 2013

Academic Editors: G. Buttazzo, B. Djafari Rouhani, and A. I. Hai

Copyright ( 2013 Jun Zhou. This is an open access article distributed under the Creative Commons Attribution License, which permits unrestricted use, distribution, and reproduction in any medium, provided the original work is properly cited.

This paper considers the singularity properties of positive solutions for a reaction-diffusion system with nonlocal boundary condition. The conditions on the existence and nonexistence of global positive solutions are given. Moreover, we establish the blow-up rate estimate for the blow-up solution.

\section{Introduction}

This paper studies the singularity properties of the following reaction-diffusion system with nonlocal boundary condition:

$$
\begin{gathered}
u_{t}=\Delta u+u^{\alpha} v^{p}, \quad v_{t}=\Delta v+u^{q} v^{\beta}, \quad x \in \Omega, t>0, \\
u(x, t)=\int_{\Omega} f(x, y) u(x, y) d y, \\
v(x, t)=\int_{\Omega} g(x, y) v(x, y) d y, \quad x \in \partial \Omega, t>0, \\
u(x, 0)=u_{0}(x), \quad v(x, 0)=v_{0}(x), \quad x \in \bar{\Omega},
\end{gathered}
$$

where $\Omega$ is a bounded domain of $\mathbb{R}^{N}, N \geq 1$, with smooth boundary $\partial \Omega$ and $\bar{\Omega}$ is the closure of $\Omega . \alpha, \beta, p$, and $q$ are positive numbers which ensure that the equations in (1) are completely coupled with the nonlinear reaction terms. The functions $f(x, y), g(x, y)$ defined for $x \in \partial \Omega, y \in \bar{\Omega}$ are nonnegative and continuous. The initial values $u_{0}(x)$ and $v_{0}(x)$ are nonnegative, which are mathematically convenient and currently followed throughout the paper. We also assume that $\left(u_{0}, v_{0}\right)$ satisfies the compatibility condition on $\partial \Omega$, and that $f(x, \cdot) \not \equiv 0$ and $g(x, \cdot) \not \equiv 0$ for any $x \in \partial \Omega$ for the sake of the meaning of nonlocal boundary.

Denote that $Q_{T}=\Omega \times(0, T), S_{T}=\partial \Omega \times(0, T)$, and $\bar{Q}_{T}=\bar{\Omega} \times[0, T)$, where $0<T \leq+\infty$. A pair of functions $(u(x, t), v(x, t))$ is called a classical solution of problem (1) if $(u, v) \in\left[C^{2,1}\left(Q_{T}\right) \cap C\left(\bar{Q}_{T}\right)\right]^{2}$ for some $T, 0<T \leq+\infty$, and satisfies (1). The local existence of classical solution of (1) is standard (see $[1,2])$. If $T<+\infty$, it is easy to see $\lim _{t \rightarrow T}\left[\max _{x \in \bar{\Omega}} u(x, t)+\max _{x \in \bar{\Omega}} v(x, t)\right]=+\infty$, and we say that the solution $(u(x, t), v(x, t))$ of problem (1) blows up at finite time $T$. If $T=+\infty,(u(x, t), v(x, t))$ is called a global solution of problem (1).

Over the past few years, many physical phenomena were formulated as nonlocal mathematical models (see $[3,4]$ ). It has also being suggested that nonlocal growth terms present a more realistic model in physics for compressible reactive gases. Problem (1) arises in the study of the heat transfer with local source $($ see $[5,6])$ and in the study of population dynamics (see $[7,8])$.

In recent years many authors have investigated the following initial boundary value problem of reaction-diffusion system:

$$
u_{t}-\triangle u=f(u, v), \quad v_{t}-\Delta v=g(u, v), \quad x \in \Omega, t>0
$$

with Dirichlet, Neumanns or Robin boundary condition, which can be used to describe heat propagation on the boundary of container (see [2, 4, 9-23] and the literatures cited therein). Specially, when $f(u, v), g(u, v)$ have the form

$$
f(u, v)=u^{\alpha} v^{p}, \quad g(u, v)=u^{q} v^{\beta},
$$

a classical result is (see $[9,10,12,20]$ ). 
Theorem A. The system (2) ( $f, g$ is of the form (3)) with homogenous Dirichlet boundary condition

$$
u(x, t)=v(x, t)=0, \quad x \in \Omega, t>0
$$

admits a unique global solution for any nonnegative initial data $u(x, t)=u_{0}(x) \not \equiv 0, v(x, t)=v_{0}(x) \neq 0$ if and only if $\alpha<1$, $\beta<1$, and $p q \leq(1-\alpha)(1-\beta)$.

However, there are some important phenomena formulated as parabolic equations which are coupled with nonlocal boundary conditions in mathematical modeling such as thermoelasticity theory (see [24-26]). In this case, the solution could be used to describe the entropy per volume of the material. The problem of nonlocal boundary conditions for linear parabolic equation of the type

$$
\begin{gathered}
u_{t}-A u=0, \quad x \in \Omega, \quad t>0, \\
u(x, t)=\int_{\Omega} f(x, y) u(y, t) d y, \quad x \in \partial \Omega, t>0, \\
u(x, 0)=u_{0}(x), \quad x \in \bar{\Omega}
\end{gathered}
$$

with uniformly elliptic operator

$$
A u=\sum_{i, j=1}^{N} a_{i, j}(x) \frac{\partial^{2}}{\partial x_{i} \partial x_{j}}+\sum_{i=1}^{N} b_{i}(x) \frac{\partial}{\partial x_{i}}+c(x)
$$

and $c(x) \leq 0$ was studied by Friedman [26]. It was proved that the unique solution of (5) tends to 0 monotonically and exponentially as $t \rightarrow+\infty$ provided $\int_{\Omega} f(x, y) d y \leq \rho<1$ for any $x \in \partial \Omega$.

As for more general discussions on the dynamic of parabolic problem with nonlocal boundary conditions, one can see Pao [27], where the following problem:

$$
\begin{gathered}
u_{t}-A u=h(x, u), \quad x \in \Omega, t>0, \\
\alpha_{0} \frac{\partial u}{\partial \nu}+u=\int_{\Omega} f(x, y) u(y, t) d y, \quad x \in \partial \Omega, t>0, \\
u(x, 0)=u_{0}(x), \quad x \in \bar{\Omega}
\end{gathered}
$$

was considered, and recently Pao [28] gave the numerical solutions for diffusion equation with nonlocal conditions.

In particular, the following single equation:

$$
\begin{gathered}
u_{t}-\Delta u=u^{\alpha}, \quad x \in \Omega, t>0, \\
u=\int_{\Omega} f(x, y) u(y, t) d y, \quad x \in \partial \Omega, t>0, \\
u(x, 0)=u_{0}(x), \quad x \in \bar{\Omega}
\end{gathered}
$$

under the assumption the $\int_{\Omega} f(x, y) d y=1$ for any $x \in \partial \Omega$ was consider, by Seo [29] and the following blow-up rate estimate is established:

$$
(\alpha-1)^{-1 /(\alpha-1)}\left(T^{*}-t\right) \leq \max _{x \in \bar{\Omega}} u(x, t) \leq C\left(T^{*}-t\right)^{-1 /(\gamma-1)},
$$

where $T^{*}<+\infty$ is the blow-up time, and $\gamma$ is any constant satisfying $1<\gamma<\alpha$.

Recently, Kong and Wang [30] obtained the blow-up conditions and blow-up profiles of the following system by using some ideas of Souplet [4]:

$$
\begin{gathered}
u_{t}=\Delta u+\int_{\Omega} u^{\alpha} v^{p} d x, \\
v_{t}=\Delta v+\int_{\Omega} u^{q} v^{\beta} d x, \quad x \in \Omega, t>0, \\
u(x, t)=\int_{\Omega} f(x, y) u(x, y) d y, \\
v(x, t)=\int_{\Omega} g(x, y) v(x, y) d y, \quad x \in \partial \Omega, t>0, \\
u(x, 0)=u_{0}(x), \quad v(x, 0)=v_{0}(x), \quad x \in \bar{\Omega} .
\end{gathered}
$$

Furthermore, Zheng and Kong [31] gave the condition for global existence or nonexistence of solution to the following system:

$$
\begin{gathered}
u_{t}=\Delta u+u^{\alpha} \int_{\Omega} v^{p} d x, \\
v_{t}=\Delta v+v^{\beta} \int_{\Omega} u^{q} d x, \quad x \in \Omega, t>0, \\
u(x, t)=\int_{\Omega} f(x, y) u(x, y) d y, \\
v(x, t)=\int_{\Omega} g(x, y) v(x, y) d y, \quad x \in \partial \Omega, t>0, \\
u(x, 0)=u_{0}(x), \quad v(x, 0)=v_{0}(x), \quad x \in \bar{\Omega} .
\end{gathered}
$$

Motivated by the above cited works, in this paper, we deal with singularity analysis of the parabolic system (1) with nonlocal boundary condition and it is seems that there is no work dealing with this type of systems except the single equations case, although this is a very classical model. Our main results read as follows.

Theorem 1. If $\alpha<1, \beta<1$, and $p q \leq(1-\alpha)(1-\beta)$, then every nonnegative solution of (1) is global.

Theorem 2. Suppose $\alpha>1$ or $\beta>1$ or pq $>(1-\alpha)(1-\beta)$.

(i) For any nonnegative functions $f(x, y)$ and $g(x, y)$, the nonnegative solution of (1) blows up in finite time provided the initial values are large enough.

(ii) If $\int_{\Omega} f(x, y) d y \geq 1$ and $\int_{\Omega} g(x, y) d y \geq 1$ for any $x \in$ $\partial \Omega$, the nonnegative solution of (1) blows up in finite time with any positive initial values.

(iii) If $\int_{\Omega} f(x, y) d y<1$ and $\int_{\Omega} g(x, y) d y<1$ for any $x \in$ $\partial \Omega$, the nonnegative solution of (1) is global with small initial values.

To estimate the blow-up rate of the blow-up solution of (1), we need to add some assumptions for initial data as follows. 
(H1) $u_{0}(x), \quad v_{0}(x) \in C^{2+\gamma}(\Omega) \cap C(\bar{\Omega})$ for some $0<\gamma<1$.

(H2) If $p-\beta \geq q-\alpha \geq 1$, then there exists a a sufficient small constant $\varepsilon_{0}>0$ (which will be given in Section 4 ) such that $\triangle u_{0}(x)+\varepsilon_{0} u_{0}^{\lambda} \geq 0$ and $\triangle v_{0}(x)+\varepsilon_{0} u_{0}^{\mu}>0$ on $\bar{\Omega}$, where

$\lambda=\frac{p(q+1)+\alpha(1-\beta)}{p-\beta+1}, \quad \mu=\frac{q(p+1)+\beta(1-\alpha)}{p-\beta+1}$.

(H3) If $q-\alpha \geq p-\beta \geq 1$, then there exists a sufficient small constant $\varepsilon_{0}>0$ (which will be given in Section 4 ) such that $\triangle u_{0}(x)+\varepsilon_{0} v_{0}^{l} \geq 0$ and $\triangle v_{0}(x)+\varepsilon_{0} v_{0}^{m}>0$ on $\bar{\Omega}$, where

$$
l=\frac{p(q+1)+\alpha(1-\beta)}{q-\alpha+1}, \quad m=\frac{q(p+1)+\beta(1-\alpha)}{q-\alpha+1} .
$$

Theorem 3. Suppose $p-\beta \geq q-\alpha \geq 1$, and $f(x, y)=g(x, y)$, $\int_{\Omega} f(x, y) d y \leq 1$ for all $x \in \partial \Omega$ and assumptions (H1)-(H2) hold. If $(u(x, t), v(x, t))$ is the smooth solution of (1) and blows up in finite time $T^{*}$, then there exist positive constants $C_{i} \quad(i=$ $1,2,3,4)$ such that

$$
\begin{aligned}
& C_{1} \leq \max _{x \in \bar{\Omega}} u(x, t)\left(T^{*}-t\right)^{(p-\beta+1) /(p q-(1-\alpha)(1-\beta))} \leq C_{2}, \\
& C_{3} \leq \max _{x \in \bar{\Omega}} v(x, t)\left(T^{*}-t\right)^{(q-\alpha+1) /(p q-(1-\alpha)(1-\beta))} \leq C_{4},
\end{aligned}
$$

for $0<t<T^{*}$.

Theorem 4. Suppose $q-\alpha \geq p-\beta \geq 1$, and $f(x, y)=g(x, y)$, $\int_{\Omega} f(x, y) d y \leq 1$ for all $x \in \partial \Omega$ and assumptions (H1) and (H3) hold. If $(u(x, t), v(x, t))$ is the smooth solution of (1) and blows up in finite time $T^{*}$, then there exist positive constants $C_{i} \quad(i=1,2,3,4)$ such that

$$
\begin{aligned}
& C_{1} \leq \max _{x \in \bar{\Omega}} u(x, t)\left(T^{*}-t\right)^{(p-\beta+1) /(p q-(1-\alpha)(1-\beta))} \leq C_{2}, \\
& C_{3} \leq \max _{x \in \bar{\Omega}} v(x, t)\left(T^{*}-t\right)^{(q-\alpha+1) /(p q-(1-\alpha)(1-\beta))} \leq C_{4},
\end{aligned}
$$

for $0<t<T^{*}$.

The rest of this paper is organized as follows. In the next section, we give some preliminaries, which include the comparison principle related to system (1). In Section 3, we will study the conditions for the solution to blowup and exist globally and prove Theorems 1 and 2. In Section 4, we will establish the precise blowup rate estimate for small weighted nonlocal boundary and prove Theorems 3 and 4 .

\section{Preliminaries}

In this section, we give some basic preliminaries. We begin with the definition of upper and lower solutions of (1).
Definition 5. A pair of nonnegative functions $(\bar{u}(x, t), \bar{v}(x, t))$ is called an upper solution of problem (1) if $(\bar{u}, \bar{v}) \in$ $\left[C^{2,1}\left(Q_{T}\right) \cap C\left(\bar{Q}_{T}\right)\right]^{2}$ and satisfies

$$
\begin{gathered}
\bar{u}_{t} \geq \triangle \bar{u}+\bar{u}^{\alpha} \bar{v}^{p}, \quad \bar{v}_{t} \geq \Delta \bar{v}+\bar{u}^{q} \bar{v}^{\beta}, \quad(x, t) \in Q_{T}, \\
\bar{u}(x, t) \geq \int_{\Omega} f(x, y) \bar{u}(x, y) d y, \\
\bar{v}(x, t) \geq \int_{\Omega} g(x, y) \bar{v}(x, y) d y, \quad(x, t) \in S_{T}, \\
\bar{u}(x, 0) \geq u_{0}(x), \quad \bar{v}(x, 0) \geq v_{0}(x), x \in \bar{\Omega} .
\end{gathered}
$$

Similarly, $(\underline{u}, \underline{v}) \in\left[C^{2,1}\left(Q_{T}\right) \cap C\left(\bar{Q}_{T}\right)\right]^{2}$ is called a lower solution of (1) if it satisfied all the reversed inequalities in (16).

Lemma 6. Let $\lambda_{i}(x, t), \Theta_{i}(x, t), i=1,2, f$, and $g$ be continuous and nonnegative functions, and let $(w(x, t), z(x, t)) \in$ $\left[C^{2,1}\left(Q_{T}\right) \cap C\left(\bar{Q}_{T}\right)\right]^{2}$ satisfy

$$
\begin{gathered}
w_{t} \geq \Delta w+\lambda_{1}(x, t) w(x, t)+\Theta_{1}(x, t) z(x, t), \quad(x, t) \in Q_{T}, \\
z_{t} \geq \Delta z+\lambda_{2}(x, t) w(x, t)+\Theta_{2}(x, t) z(x, t), \quad(x, t) \in Q_{T}, \\
w(x, t) \geq \int_{\Omega} f(x, y) w(x, y) d y, \\
z(x, t) \geq \int_{\Omega} g(x, y) z(x, y) d y, \quad(x, t) \in S_{T}, \\
w(x, 0) \geq 0, \quad z(x, 0) \geq 0, \quad x \in \bar{\Omega},
\end{gathered}
$$

and then $w(x, t)>0, z(x, t)>0$ on $\bar{Q}_{T}$.

Proof. Set $t_{1}=\sup \{t \in(0, T): w(x, t)>0, z(x, t)>0, x \in$ $\bar{\Omega}\}$. Since $w(x, 0)>0, z(x, 0)>0$, by continuous, there exists $\delta>0$ such that $w(x, t)>0, z(x, t)>0$ for all $(x, t) \in \bar{\Omega} \times$ $[0, \delta)$. Thus, $t_{1} \in(\delta, T]$.

We claim that $t_{1}<T$ will lead to a contradiction. Indeed, $t_{1}<T$ suggests that $w\left(x_{1}, t_{1}\right)=0$ or $z\left(x_{1}, t_{1}\right)=0$ for some $x_{1} \in \bar{\Omega}$. Without loss of generality, we suppose that $w\left(x_{1}, t_{1}\right)=0=\inf _{(x, t) \in \overline{\mathrm{Q}}_{t_{1}}} w(x, t)$.

If $x_{1} \in \Omega$, we first notice that

$$
\begin{aligned}
w_{t}-\Delta w \geq & \lambda_{1}(x, t) w(x, t) \\
& +\Theta_{1}(x, t) z(x, t) \geq 0, \quad(x, t) \in Q_{t_{1}} .
\end{aligned}
$$

In addition, it is clear that $w \geq 0$ on the boundary $\partial \Omega$ and at initial state $t=0$. Then it follows from the strong maximum principle that $w \equiv 0$ in $Q_{t_{1}}$, which contradicts $w(x, 0)>0$.

If $x_{1} \in \partial \Omega$, we will have a contradiction

$$
0=w\left(x_{1}, t_{1}\right) \geq \int_{\Omega} f\left(x_{1}, y\right) w\left(y, t_{1}\right)>0 .
$$

In the last inequality, we have used the facts that $f(x, \cdot) \not \equiv$ for any $x \in \partial \Omega$ and $w\left(y, t_{1}\right)>0$ for any $y \in \Omega$, which is a direct result of the previous case.

Therefore, the claim is true and thus $t_{1}=T$, which implies that $w>0, z>0$ on $\bar{Q}_{T}$. The proof is complete. 
Remark 7. If $\int_{\Omega} f(x, y) d y \leq 1$ and $\int_{\Omega} g(x, y) d y \leq 1$ for any $x \in \partial \Omega$ in Lemma 6 , we can obtain $w(x, t) \geq 0, z(x, t) \geq$ 0 on $\bar{Q}_{T}$ under the assumption that $w(x, 0) \geq 0, z(x, 0) \geq$ 0 for any $x \in \bar{\Omega}$. Indeed, for any $\epsilon>0$, we can conclude that $\left(w(x, t)+\epsilon e^{t}, z(x, t)+\epsilon e^{t}\right)>(0,0)$ on $\bar{Q}_{T}$ as the proof of Lemma 6 . Then the desired result follows from the limit procedure $\epsilon \rightarrow 0$.

Lemma 8. Let $(\bar{u}(x, t), \bar{v}(x, t))$ and $(\underline{u}(x, t), \underline{v}(x, t))$ be a upper and lower solution of (1) in $Q_{T}$, respectively. If $(\bar{u}(x, 0), \bar{v}(x$, $0))>(\underline{u}(x, 0), \underline{v}(x, 0))$ for $x \in \bar{\Omega}$, then $(\bar{u}(x, t), \bar{v}(x, t))>(\underline{u}(x$, $t), \underline{v}(x, t))$ on $\bar{Q}_{T}$.

Proof. Let $w(x, t)=\bar{u}(x, t)-\underline{u}(x, t)$ and $z(x, t)=\bar{v}(x, t)-$ $\underline{v}(x, t)$, and then

$$
\begin{aligned}
w_{t}-\triangle w \geq & \bar{u}^{\alpha} \bar{v}^{p}-\underline{u}^{\alpha} \underline{v}^{p}=\bar{v}^{p}\left(\bar{u}^{\alpha}-\underline{u}^{\alpha}\right)+\underline{u}^{\alpha}\left(\bar{v}^{p}-\underline{v}^{p}\right) \\
= & \bar{v}^{p} \phi_{1} w+\underline{u}^{\alpha} \phi_{2} z:=\lambda_{1}(x, t) w(x, t) \\
& +\Theta_{1}(x, t) z(x, t), \\
z_{t}-\triangle z \geq & \bar{u}^{q} \bar{v}^{\beta}-\underline{u}^{q} \underline{v}^{\beta} \\
= & \bar{v}^{\beta}\left(\bar{u}^{q}-\underline{u}^{q}\right)+\underline{u}^{q}\left(\bar{v}^{\beta}-\underline{v}^{\beta}\right) \\
= & \bar{v}^{\beta} \phi_{3} w+\underline{u}^{q} \phi_{4} z:=\lambda_{2}(x, t) w(x, t) \\
& +\Theta_{2}(x, t) z(x, t),
\end{aligned}
$$

where

$$
\begin{aligned}
& \lambda_{1}(x, t)=\bar{v}^{p} \phi_{1}=\bar{v}^{p} \int_{0}^{1} \alpha(\lambda \bar{u}+(1-\lambda) \underline{u})^{\alpha-1} d \lambda, \\
& \lambda_{2}(x, t)=\bar{v}^{\beta} \phi_{3}=\bar{v}^{\beta} \int_{0}^{1} q(\lambda \bar{u}+(1-\lambda) \underline{u})^{q-1} d \lambda, \\
& \Theta_{1}(x, t)=\underline{u}^{\alpha} \phi_{2}=\underline{u}^{\alpha} \int_{0}^{1} p(\lambda \bar{v}+(1-\lambda) \underline{v})^{p-1} d \lambda, \\
& \Theta_{2}(x, t)=\underline{u}^{q} \phi_{4}=\underline{u}^{q} \int_{0}^{1} \beta(\lambda \bar{v}+(1-\lambda) \underline{v})^{\beta-1} d \lambda .
\end{aligned}
$$

So, the functions $w(x, t)$ and $z(x, t)$ satisfy

$$
\begin{gathered}
w_{t} \geq \Delta w+\lambda_{1}(x, t) w(x, t)+\Theta_{1}(x, t) z(x, t), \quad(x, t) \in Q_{T} \\
z_{t} \geq \Delta z+\lambda_{2}(x, t) w(x, t)+\Theta_{2}(x, t) z(x, t), \quad(x, t) \in Q_{T} \\
w(x, t) \geq \int_{\Omega} f(x, y) w(x, y) d y, \\
z(x, t) \geq \int_{\Omega} g(x, y) z(x, y) d y, \quad(x, t) \in S_{T} \\
w(x, 0) \geq 0, \quad z(x, 0) \geq 0, \quad x \in \bar{\Omega} .
\end{gathered}
$$

Lemma 6 ensures that $w(x, t)>0, z(x, t)>0$ on $\bar{Q}_{T}$, that is, $(\bar{u}(x, t), \bar{v}(x, t))>(\underline{u}(x, t), \underline{v}(x, t))$ on $\bar{Q}_{T}$. The proof is complete.

\section{Global Existence and Blowup}

In this section, we will use the upper and lower solutions and their corresponding comparison principle developed in Section 2 to get the global existence or finite blowup of the solution to (1). Let us first give the proof of Theorem 1 .

Proof of Theorem 1. Using the condition $\alpha<1, \beta<1, p q \leq$ $(1-\alpha)(1-\beta)$, and $p>0, q>0$, we have $((1-\alpha) / p) \cdot((1-$ $\beta) / q) \geq 1$. Thus, we can choose two positive constant and $m, l>1$ such that

$$
\frac{1-\alpha}{p} \geq \frac{l}{m}, \quad \frac{1-\beta}{q} \geq \frac{m}{l} .
$$

Then, let $\phi(x, y)(x \in \partial \Omega, y \in \bar{\Omega})$ be a continuous function such that $\phi(x, y) \geq \max \{f(x, y), g(x, y)\}$ and set

$$
\begin{gathered}
a(x)=\left[\int_{\Omega} \phi(x, y) d y\right]^{(1-m) / m}, \\
b(x)=\left[\int_{\Omega} \phi(x, y) d y\right]^{(1-l) / l}, \quad x \in \partial \Omega .
\end{gathered}
$$

We consider the following auxiliary problem:

$$
\begin{gathered}
w_{t}=\Delta w+k w, \quad x \in \Omega, t>0, \\
w(x, t)=(a(x)+b(x)+1) \\
\times\left[\int_{\Omega}\left(\phi(x, y)+\frac{1}{|\Omega|}\right) w(y, t) d y\right], \\
x \in \partial \Omega, t>0, \\
w(x, 0)=1+u_{0}^{1 / m}(x)+v_{0}^{1 / l}(x), \quad x \in \bar{\Omega},
\end{gathered}
$$

where $|\Omega|$ is the measure of $\Omega$ and $k=(1 / m)+(1 / l)$. It follows from [32, Theorem 4.2] that $w(x, t)$ exists globally and indeed $w(x, t)>1$ on $\bar{\Omega} \times[0,+\infty)[32$, Theorem 2.1].

Our aim is to show that $(\bar{u}, \bar{v})=\left(w^{m}, w^{l}\right)$ is a global upper solution of (1). Indeed, a direct computation yields

$$
\begin{aligned}
\bar{u}_{t}-\Delta \bar{u} \geq w^{m} & =\left(w^{m}\right)^{\alpha} w^{m(1-\alpha)} \\
& =\bar{u}^{\alpha}\left(w^{l}\right)^{m(1-\alpha) / l} \geq \bar{u}^{\alpha} \bar{v}^{p}, \quad x \in \Omega, t>0 .
\end{aligned}
$$

Here, we have used that conclusion $w>1$ and the inequality (23). We still have to consider the boundary and initial conditions. When $x \in \partial \Omega$, we have

$$
\begin{aligned}
\bar{u}(x, t)= & (a(x)+b(x)+1)^{m} \\
& \times\left[\int_{\Omega}\left(\phi(x, y)+\frac{1}{|\Omega|}\right) w(y, t) d y\right]^{m}
\end{aligned}
$$




$$
\begin{aligned}
\geq & (a(x))^{m}\left[\int_{\Omega} \phi(x, y) w(y, t) d y\right]^{m} \\
= & {\left[\int_{\Omega} \phi(x, y) d y\right]^{1-m}\left[\int_{\Omega} \phi(x, y) w(y, t) d y\right]^{m} } \\
\geq & {\left[\int_{\Omega} f(x, y) d y\right]^{1-m}\left[\int_{\Omega} f(x, y) w(y, t) d y\right]^{m} } \\
= & {\left[\int_{\Omega}\left(f^{1-m}(x, y)\right)^{1 /(1-m)} d y\right]^{1-m} } \\
& \times\left[\int_{\Omega}\left(f^{m}(x, y) w^{m}(y, t)\right)^{1 / m} d y\right]^{m} \\
\geq & \int_{\Omega} f^{1-m}(x, y)(f(x, y) w(x, y))^{m} d y \\
= & \int_{\Omega} f(x, y) w^{m}(x, y) d y \\
= & \int_{\Omega} f(x, y) \bar{u}(x, y) d y, \quad x \in \partial \Omega, t>0 .
\end{aligned}
$$

Similarly, we have

$$
\begin{gathered}
\bar{v}_{t}-\Delta \bar{v} \geq \bar{u}^{q} v^{\beta}, \quad x \in \Omega, t>0, \\
\bar{v} \geq \int_{\Omega} g(x, t) \bar{v}(y, t) d y, \quad x \in \partial \Omega, t>0 .
\end{gathered}
$$

It is clear that $u_{0}(x)<\bar{u}(x, 0)$ and $v_{0}(x)<\bar{v}(x, 0)$. Therefore, we get that $(\bar{u}, \bar{v})$ is a global upper solution of (1) and hence the solution of (1) exists globally by Lemma 8 . The proof is complete.

Proof of Theorem 2. (i) Let $(u, v)$ be the solution of (2) and (3) with homogeneous Dirichlet boundary. Then it is well known for sufficiently large initial data that the solution $(\underline{u}, \underline{v})$ blows up in finite time when $\alpha>1$ or $\beta>1$ or $p q>(1-\alpha)(1-\beta)$ (Theorem A). On the other hand, it is obvious that $(\underline{u}, \underline{v})$ is a lower solution of (1). Hence, the solution of (1) with large initial data blows up in finite time.

(ii) We consider the following ODE system:

$$
\begin{gathered}
s^{\prime}(t)=s^{\alpha} h^{p}, \quad h^{\prime}(t)=s^{q} h^{\beta}, \quad t>0, \\
s(0)=a=\frac{1}{2} \min _{x \in \bar{\Omega}} u_{0}(x), \quad h(0)=b=\frac{1}{2} \min _{x \in \bar{\Omega}} v_{0}(x) .
\end{gathered}
$$

If $\alpha>1$ or $\beta>1$, it is clear that the solution $(s(t), h(t))$ of (29) blows up in finite time. For the case $0<\alpha, \beta<1$ and $p q>(1-\alpha)(1-\beta)$, it follows that

$$
\begin{aligned}
& \frac{1}{q-\alpha+1}\left[s^{q-\alpha+1}(t)-a^{q-\alpha+1}\right] . \\
& \quad=\frac{1}{p-\beta+1}\left[h^{p-\beta+1}(t)-b^{p-\beta+1}\right] .
\end{aligned}
$$

Thus, we get

$$
\begin{gathered}
h^{\prime}(t)=\left[\frac{q-\alpha+1}{p-\beta+1} h^{p-\beta+1}\right. \\
\left.+\left(a^{q-\alpha+1}-\frac{q-\alpha+1}{p-\beta+1} b^{p-\beta+1}\right)\right]^{q /(p-\beta+1)} h^{\beta} \\
\quad t>0, \\
h(0)=b>0 .
\end{gathered}
$$

Then $p q>(1-\alpha)(1-\beta)$ implies that $h(t)$ blows up in finite time, and so does $f(t)$. From the above analysis, we see that $\alpha>1$ or $\beta>1$ or $p q>(1-\alpha)(1-\beta)$ implies that $(s, h)$ blows up in finite time. Under the assumption $\int_{\Omega} f(x, y) d y \geq 1$ and $\int_{\Omega} g(x, y) d y \geq 1$ for any $x \in \partial \Omega,(s, h)$ is a lower solution of problem (1). Therefore, by Lemma 8 , we see that the solution $(u, v)$ of $(1)$ satisfies $(u, v) \geq(s, h)$ and then $(u, v)$ blows up in finite time.

(iii) Let $\psi_{1}(x)$ be the positive solution of the linear elliptic problem

$$
-\Delta \psi_{1}(x)=\varepsilon_{0}, \quad x \in \Omega,
$$

$$
\psi_{1}(x)=\int_{\Omega} f(x, y) d y, \quad x \in \partial \Omega,
$$

and $\psi_{2}(x)$ be the positive solution of the linear elliptic problem

$$
\begin{gathered}
-\Delta \psi_{2}(x)=\varepsilon_{0}, \quad x \in \Omega, \\
\psi_{2}(x)=\int_{\Omega} g(x, y) d y, \quad x \in \partial \Omega .
\end{gathered}
$$

Since $\int_{\Omega} f(x, y) d y<1$ and $\int_{\Omega} g(x, y) d y<1$ for any $x \in \partial \Omega$, we can choose $\varepsilon_{0}>0$ such that $0 \leq \psi_{i}(x) \leq 1, i=1,2$.

Let $\bar{u}(x)=a \psi_{1}(x)$ and $\bar{v}(x)=b \psi_{2}(x)$, where $a, b$ are positive constants which satisfy $a \varepsilon_{0} \geq a^{\alpha} b^{p}, b \varepsilon_{0} \geq a^{q} b^{\beta}$. We remark that under the assumption $\alpha>1$ or $\beta>1$ or $p q>(1-$ $\alpha)(1-\beta)$, we can choose such $a, b$ easily. We now show that $(\bar{u}, \bar{v})$ is an upper solution of (1) for small initial data $\left(u_{0}, v_{0}\right)$. Indeed, for any $x \in \Omega$, we have

$$
\begin{aligned}
& \bar{u}_{t}-\triangle \bar{u}=a \varepsilon_{0} \geq a^{\alpha} b^{p} \geq \bar{u}^{\alpha} \bar{v}^{p}, \\
& \bar{v}_{t}-\triangle \bar{v}=b \varepsilon_{0} \geq a^{q} b^{\beta} \geq \bar{u}^{q} \bar{v}^{\beta} .
\end{aligned}
$$

When $x \in \partial \Omega$,

$$
\begin{aligned}
\bar{u}(x) & =a \int_{\Omega} f(x, y) d y \geq \int_{\Omega} f(x, y) a \psi_{1}(y) d y \\
& =\int_{\Omega} f(x, y) \bar{u}(y) d y \\
\bar{v}(x) & =b \int_{\Omega} g(x, y) d y \geq \int_{\Omega} g(x, y) b \psi_{2}(y) d y \\
& =\int_{\Omega} g(x, y) \bar{v}(y) d y .
\end{aligned}
$$

Here, we have used $\psi_{i} \leq 1, i=1,2$. The above inequalities show that $(\bar{u}, \bar{v})$ is an upper solution of (1) whenever $u_{0}(x)<$ $a \psi_{1}(x)$ and $v_{0}(x)<b \psi_{2}(x)$. The proof is complete. 


\section{Blowup Rate}

In this section, we will estimate the blow-up rate of (1). By the standard methods (see $[1,2,6])$, we can show that system (1) has a smooth solution $(u, v)$ provided that $u_{0}, v_{0}$ satisfy the hypotheses (H1). We thus assume that the smooth solution $(u, v)$ of (1) blows up at finite time $T^{*}$ and set $M_{1}(t)=$ $\max _{x \in \bar{\Omega}} u(x, t), M_{2}(t)=\max _{x \in \bar{\Omega}} v(x, t)$. We can obtain the blow-up rate from the following lemmas.

Lemma 9. Suppose that $u_{0}(x), v_{0}(x)$ satisfy (H1), and then there exists a positive constant $K_{1}$ such that

$$
\begin{aligned}
& M_{1}^{q-\alpha+1}(t)+M_{2}^{p-\beta+1}(t) \\
& \quad \geq K_{1}\left(T^{*}-t\right)^{-(q-\alpha+1)(p-\beta+1) /(p q-(1-\alpha)(1-\beta))} .
\end{aligned}
$$

Proof. By the equations in (1), we have [33, Theorem 4.5]

$$
M_{1}^{\prime} \leq M_{1}^{\alpha} M_{2}^{p}, \quad M_{2}^{\prime} \leq M_{1}^{q} M_{2}^{\beta}, \quad \text { a.e. }
$$

Noticing that $q-\alpha+1>0$ and $p-\beta+1>0$, we have

$$
\begin{aligned}
\left(M_{1}^{q-\alpha+1}(t)\right. & \left.+M_{2}^{p-\beta+1}(t)\right)^{\prime} \\
\leq & (p+q-\alpha-\beta+2) M_{1}^{\beta}(t) M_{2}^{\alpha}(t) \\
\leq & K_{2}\left(M_{1}^{q-\alpha+1}(t)\right. \\
& \left.\quad+M_{2}^{p-\beta+1}(t)\right)^{((q-\alpha+1) q+(p-\beta+1) p) /((q-\alpha+1)(p-\beta+1))}
\end{aligned}
$$

by virtue of Young's inequality. Integrating (38) from $t$ to $T^{*}$, we can get (36). The proof is complete.

Lemma 10. If $p-\beta \geq q-\alpha \geq 1, f(x, y)=g(x, y)$, and $\int_{\Omega} f(x, y) d y \leq 1$ for any $x \in \partial \Omega$, then there exists a positive constant $c_{0}$ such that the solution $(u, v)$ of (1) with positive initial value $\left(u_{0}, v_{0}\right)$ satisfies

$$
u(x, t) \geq c_{0} v^{(p-\beta+1) /(q-\alpha+1)}(x, t), \quad(x, t) \in \Omega \times\left[0, T^{*}\right) .
$$

Proof. Let $J(x, t)=u(x, t)-c_{0} v^{(p-\beta+1) /(q-\alpha+1)}(x, t)$, where $c_{0}$ is a positive constant to be chosen. For $(x, t) \in \Omega \times\left(0, T^{*}\right)$, a series of calculations shows that

$$
\begin{aligned}
J_{t}-\triangle & \\
= & u_{t}-c_{0} \frac{p-\beta+1}{q-\alpha+1} v^{(p-q+\alpha-\beta) /(q-\alpha+1)} v_{t}-\triangle u \\
& +c_{0} \frac{(p-\beta+1)(p-q+\alpha-\beta)}{q-\alpha+1} v^{(p-2 \alpha-2 q-\beta-1) /(q-\alpha+1)}
\end{aligned}
$$

$$
\begin{aligned}
& \times|\nabla u|^{2}+c_{0} \frac{p-\beta+1}{q-\alpha+1} v^{(p-q+\alpha-\beta) /(q-\alpha+1)} \Delta v \\
\geq & u^{\alpha} v^{p}-c_{0} \frac{p-\beta+1}{q-\alpha+1} v^{(p-q+\alpha-\beta) /(q-\alpha+1)} u^{q} v^{\beta} \\
= & u^{\alpha} v^{\beta+(p-q+\alpha-\beta) /(q-\alpha+1)} \\
& \times\left[v^{(p-\beta+1)(q-\alpha) /(q-\alpha+1)}-c_{0} \frac{p-\beta+1}{q-\alpha+1} u^{q-\alpha}\right] \\
= & u^{\alpha} v^{\beta+(p-q+\alpha-\beta) /(q-\alpha+1)} \\
& \times\left[c_{0}^{\alpha-q}(u-J)^{q-\alpha}-c_{0} \frac{p-\beta+1}{q-\alpha+1} u^{q-\alpha}\right] .
\end{aligned}
$$

If we choose $c_{0} \leq[(q-\alpha+1) /(p-\beta+1)]^{1 /(q-\alpha+1)}$, then $c_{0}^{\alpha-q} \geq c_{0}((p-\beta+1) /(q-\alpha+1))$. So, we have

$$
\begin{gathered}
J_{t}-\Delta J+c_{0}(q-\alpha) \frac{p-\beta+1}{q-\alpha+1} u^{\alpha} \\
v^{\beta+(p-q+\alpha-\beta) /(q-\alpha+1)} \theta^{q-\alpha+1}(u, v) J \geq 0,
\end{gathered}
$$

where $\theta(u, v)$ is a function of $u$ and $v$, which lines between $u-J$ and $u$.

When $(x, t) \in \partial \Omega \times\left(0, T^{*}\right)$, we have

$$
\begin{aligned}
J(x, t)= & \int_{\Omega} f(x, y) u(y, t) d y \\
& -c_{0}\left[\int_{\Omega} f(x, y) v(y, t) d y\right]^{(p-\beta+1) /(q-\alpha+1)} .
\end{aligned}
$$

Denote that $H(x)=\int_{\Omega} f(x, y) d y \geq 0$ for any $x \in \partial \Omega$. Since $f(x, \cdot) \not \equiv 0$ for any $x \in \partial \Omega, H(x)>0$. It follows from Jensen's inequality $H(x) \leq 1$ and $(p-\beta+1) /(q-\alpha+1) \geq 1$ that

$$
\begin{aligned}
\int_{\Omega} f(x, y) v^{(p-\beta+1) /(q-\alpha+1)}(y, t) d y & \\
- & {\left[\int_{\Omega} f(x, y) v(y, t) d y\right]^{(p-\beta+1) /(q-\alpha+1)} } \\
\geq & H(x)\left[\int_{\Omega} f(x, y) \frac{v(y, t)}{H(x)} d y\right]^{(p-\beta+1) /(q-\alpha+1)} \\
& -\left[\int_{\Omega} f(x, y) v(y, t) d y\right]^{(p-\beta+1) /(q-\alpha+1)} \geq 0 .
\end{aligned}
$$

Combining the above inequality with (42), we obtain

$$
\begin{aligned}
J(x, t) \geq & \int_{\Omega} f(x, y) u(y, t) d y \\
& -c_{0} \int_{\Omega} f(x, y) v^{(p-\beta+1) /(q-\alpha+1)}(y, t) d y \\
= & \int_{\Omega} f(x, y) J(y, t) d y .
\end{aligned}
$$


For the initial condition, we have

$$
J(x, 0)=u_{0}(x)-c_{0} v_{0}^{(p-\beta+1) /(q-\alpha+1)}(x) \geq 0
$$

on $\bar{\Omega}$ provided that $c_{0} \leq \inf _{x \in \bar{\Omega}} u_{0}(x) v_{0}^{-(p-\beta+1) /(q-\alpha+1)}(x)$.

Summarily, if we take $c_{0}$ small enough such that

$$
\begin{gathered}
\mathcal{c}_{0} \leq \min \left\{\inf _{x \in \bar{\Omega}} u_{0}(x) v_{0}^{-(p-\beta+1) /(q-\alpha+1)}(x),\right. \\
\left.\left[\frac{q-\alpha+1}{p-\beta+1}\right]^{1 /(q-\alpha+1)}\right\},
\end{gathered}
$$

it follows from (41), (44), (45), and [32, Theorem 2.1] that $J(x, t) \geq 0$, which implies (39). The proof is complete.

Combining (39) with (1), we know that the solution $(u, v)$ of (1) satisfies

$$
\begin{gathered}
u_{t} \leq \Delta u+a u^{\lambda}, \quad v_{t} \leq \Delta v+b v^{\mu}, \quad x \in \Omega, 0<t<T^{*}, \\
u(x, t)=\int_{\Omega} f(x, y) u(x, y) d y, \\
v(x, t)=\int_{\Omega} g(x, y) v(x, y) d, \quad x \in \partial \Omega, 0<t<T^{*}, \\
u(x, 0)=u_{0}(x), \quad v(x, 0)=v_{0}(x), \quad x \in \bar{\Omega}
\end{gathered}
$$

if $f(x, y)=g(x, y)$, where $a=\left[1 / c_{0}\right]^{(p(q-\alpha+1)) /(p-\beta+1)}, b=$ $\left[1 / c_{0}\right]^{(\beta(q-\alpha+1)) /(p-\beta+1)}, \lambda=(p(q+1)+\alpha(1-\beta)) /(p-\beta+1)$, and $\mu=(q(p+1)+\beta(1-\alpha)) /(p-\beta+1)$. It is easy to see that $\lambda>1$ and $\mu>1$ if $p-\beta \geq q-\alpha \geq 1$.

Let $(w, z)$ be the solution of the following system:

$$
\begin{gathered}
w_{t}=\Delta w+a w^{\lambda}, \quad z_{t}=\Delta z+b w^{\mu}, \quad x \in \Omega, 0<t<T^{*}, \\
w(x, t)=\int_{\Omega} f(x, y) w(x, y) d y, \\
z(x, t)=\int_{\Omega} f(x, y) z(x, y) d y, \quad x \in \partial \Omega, 0<t<T^{*}, \\
w(x, 0)=u_{0}(x), \quad z(x, 0)=v_{0}(x), \quad x \in \bar{\Omega} .
\end{gathered}
$$

It is easy to see that $(w, z) \geq(u, v)$ by Remark 7 if $\int_{\Omega} f(x$, $y) d y \leq 1$ for any $x \in \partial \Omega$.

Lemma 11. Suppose that $u_{0}(x)$ and $v_{0}(x)$ satisfy $(H 1)-(H 2)$ and that the assumptions in Lemma 10 hold; then the solution $(w, z)$ of (48) satisfies

$$
w_{t}-a \delta w^{\lambda} \geq 0, \quad z_{t}-b \delta w^{\mu} \geq 0, \quad(x, t) \in \Omega \times\left(0, T^{*}\right)
$$

if $\varepsilon_{0}$ is small enough such that $0<\varepsilon_{0}<\min \{a, b\}$ and $\delta \leq$ $\min \left\{1-\varepsilon_{0} / a, 1-\varepsilon_{0} / b\right\}$.
Proof. Let $J_{1}(x, t)=w_{t}-a \delta w^{\lambda}$ and $J_{2}(x, t)=z_{t}-b \delta w^{\mu}$. For $(x, t) \in \Omega \times\left(0, T^{*}\right)$, a series of calculations shows that

$$
J_{1 t}-\triangle J_{1} \geq a \lambda w^{\lambda-1} J_{1}, \quad J_{2 t}-\triangle J_{2} \geq b \mu w^{\mu-1} J_{1} .
$$

For $(x, t) \in \partial \Omega \times\left(0, T^{*}\right)$, using the boundary conditions we have

$$
\begin{aligned}
J_{1}(x, t)= & w_{t}-a \delta w^{\lambda} \\
= & \int_{\Omega} f(x, y) w_{t}(y, t) d y \\
& -a \delta\left[\int_{\Omega} f(x, y) w(y, t) d y\right]^{\lambda} \\
= & \int_{\Omega} f(x, y)\left(J_{1}+a \delta w^{\lambda}\right)(y, t) d y \\
& -a \delta\left[\int_{\Omega} f(x, y) w(y, t) d y\right]^{\lambda} \\
= & \int_{\Omega} f(x, y) J_{1}(x, y) d y \\
& +a \delta\left[\int_{\Omega} f(x, y) w^{\lambda}(y, t) d y\right. \\
& \left.-\left[\int_{\Omega} f(x, y) w(y, t) d y\right]^{\lambda}\right] .
\end{aligned}
$$

It follows from $\int_{\Omega} f(x, y) d y \leq 1$ for any $x \in \partial \Omega$ and Jensen's inequality that

$$
\int_{\Omega} f(x, y) w^{\lambda}(y, t) d y \geq\left[\int_{\Omega} f(x, y) w(y, t) d y\right]^{\lambda} .
$$

Thus,

$$
J_{1}(x, t) \geq \int_{\Omega} f(x, y) J_{1}(x, y) d y, \quad(x, t) \in \partial \Omega \times\left(0, T^{*}\right) .
$$

Similarly, we have

$$
J_{2}(x, t) \geq \int_{\Omega} f(x, y) J_{2}(x, y) d y, \quad(x, t) \in \partial \Omega \times\left(0, T^{*}\right) .
$$

For the initial condition, under assumption (H2), we have

$$
J_{1}(x, 0) \geq 0, \quad J_{2}(x, t) \geq 0, \quad x \in \bar{\Omega}
$$

if $\varepsilon_{0}$ is small enough such that $0<\varepsilon_{0}<\min \{a, b\}$ and $\delta \leq$ $\min \left\{1-\varepsilon_{0} / a, 1-\varepsilon_{0} / b\right\}$. Then (49) follows from (50)-(55). The proof is complete.

Proof of Theorem 3. Integrating the inequality for $w$ in (49) on $\left[t, T^{*}\right)$ yields

$$
w(x, t) \leq c_{1}\left(T^{*}-t\right)^{1 /(1-\lambda)}, \quad(x, t) \in \bar{\Omega} \times\left[0, T^{*}\right),
$$


where $c_{1}=(a \delta(\lambda-1))^{1 /(1-\lambda)}$. Since $u(x, t) \leq w(x, t)$, we obtain

$$
M_{1}(t) \leq c_{1}\left(T^{*}-t\right)^{1 /(1-\lambda)}=c_{1}\left(T^{*}-t\right)^{-(p-\beta+1) /(p q-(1-\alpha)(1-\beta))} .
$$

Combining (36) and (57), we get

$$
C_{1} \leq \max _{x \in \bar{\Omega}} u(x, t)\left(T^{*}-t\right)^{(p-\beta+1) /(p q-(1-\alpha)(1-\beta))} \leq C_{2},
$$

where $C_{1}$ and $C_{2}$ are two positive constants.

Since $M_{2}^{\prime} \leq M_{1}^{q} M_{2}^{\beta}$, it follows from (57) that

$$
M_{2}^{-\beta} M_{2}^{\prime} \leq c_{1}^{q}\left(T^{*}-t\right)^{-q(p-\beta+1) /(p q-(1-\alpha)(1-\beta))} .
$$

Integrating this inequality from 0 to $t$, we get

$$
M_{2}(t) \leq c_{2}\left(T^{*}-t\right)^{-(q-\alpha+1) /(p q-(1-\alpha)(1-\beta))},
$$

where $c_{2}$ is a positive constant. Combining (36) and (60), we get

$$
C_{3} \leq \max _{x \in \bar{\Omega}} v(x, t)\left(T^{*}-t\right)^{(q-\alpha+1) /(p q-(1-\alpha)(1-\beta))} \leq C_{4},
$$

where $C_{3}$ and $C_{4}$ are two positive constants. We completed the proof of Theorem 3 .

Proof of Theorem 4. The proof is similar to the proof of Theorem 3, and so we omit it.

\section{Acknowledgments}

This study is partially supported by the NSFC Grant 11201380 , the Fundamental Research Funds for the Central Universities Grant XDJK2012B007, Doctor Fund of Southwest University Grant SWU111021, and Educational Fund of Southwest University Grant 2010JY053.

\section{References}

[1] O. A. Ladyženskaja, V. A. Solonik, and N. N. Ural'ceva, Linear and Quasilinear Equations of Parabolic Type, vol. 23 of Translations of Mathematical Monographs, American Mathematical Society, Providence, RI, USA, 1967.

[2] C. V. Pao, Nonlinear Parabolic and Elliptic Equations, Plenum Press, New York, NY, USA, 1992.

[3] K. Bimpong-Bota, P. Ortoleva, and J. Ross, "Far-fromequilibrium phenomena at local sites of reaction," The Journal of Chemical Physics, vol. 60, no. 8, pp. 3124-3133, 1974.

[4] P. Souplet, "Uniform blow-up profiles and boundary behavior for diffusion equations with nonlocal nonlinear source," Journal of Differential Equations, vol. 153, no. 2, pp. 374-406, 1999.

[5] V. A. Galaktionov, "On asymptotic self-similar behaviour for a quasilinear heat equation: single point blow-up," SIAM Journal on Mathematical Analysis, vol. 26, no. 3, pp. 675-693, 1995.

[6] A. A. Samarskii, V. A. Galaktionov, S. P. Kurdyumov, and A. P. Mikhailoi, Blow-Up in Quasilinear Parabolic Equations, Nauka, Moscow, Russia, 1987.
[7] R. S. Cantrell and C. Cosner, "Diffusive logistic equations with indefinite weights: population models in disrupted environments. II," SIAM Journal on Mathematical Analysis, vol. 22, no. 4, pp. 1043-1064, 1989.

[8] J. Furter and M. Grinfeld, "Local vs. nonlocal interactions in population dynamics," Journal of Mathematical Biology, vol. 27, no. 1, pp. 65-80, 1989.

[9] J. M. Chadam, A. Peirce, and H.-M. Yin, "The blowup property of solutions to some diffusion equations with localized nonlinear reactions," Journal of Mathematical Analysis and Applications, vol. 169, no. 2, pp. 313-328, 1992.

[10] H. Chen, "Global existence and blow-up for a nonlinear reaction-diffusion system," Journal of Mathematical Analysis and Applications, vol. 212, no. 2, pp. 481-492, 1997.

[11] F. Dickstein and M. Escobedo, "A maximum principle for semilinear parabolic systems and applications," Nonlinear Analysis: Theory, Methods \& Applications, vol. 45, no. 7, pp. 825-837, 2001.

[12] M. Escobedo and M. A. Herrero, "A semilinear parabolic system in a bounded domain," Annali di Matematica Pura ed Applicata, vol. 165, pp. 315-336, 1993.

[13] V. A. Galaktionov and J. L. Vázquez, "The problem of blowup in nonlinear parabolic equations," Discrete and Continuous Dynamical Systems A, vol. 8, no. 2, pp. 399-433, 2002.

[14] F.-C. Li, S.-X. Huang, and C.-H. Xie, "Global existence and blow-up of solutions to a nonlocal reaction-diffusion system," Discrete and Continuous Dynamical Systems A, vol. 9, no. 6, pp. 1519-1532, 2003.

[15] L. Gang and B. D. Sleeman, "Non-existence of global solutions to systems of semi-linear parabolic equations," Journal of Differential Equations, vol. 104, no. 1, pp. 147-168, 1993.

[16] F. Merle and H. Zaag, "O.D.E. type behavior of blow-up solutions of nonlinear heat equations," Discrete and Continuous Dynamical Systems A, vol. 8, no. 2, pp. 435-450, 2002.

[17] J. D. Rossi and N. Wolanski, "Blow-up vs. global existence for a semilinear reaction-diffusion system in a bounded domain," Communications in Partial Differential Equations, vol. 20, no. 11-12, pp. 1991-2004, 1995.

[18] P. Souplet, "Blow-up in nonlocal reaction-diffusion equations," SIAM Journal on Mathematical Analysis, vol. 29, no. 6, pp. 13011334, 1998.

[19] M. Wang and Y. Wang, "Properties of positive solutions for non-local reaction-diffusion problems," Mathematical Methods in the Applied Sciences, vol. 19, no. 14, pp. 1141-1156, 1996.

[20] M. Wang, "Global existence and finite time blow up for a reaction-diffusion system," Zeitschrift für Angewandte Mathematik und Physik, vol. 51, no. 1, pp. 160-167, 2000.

[21] J. Zhang, "Boundedness and blow-up behavior for reactiondiffusion systems in a bounded domain," Nonlinear Analysis: Theory, Methods \& Applications, vol. 35, no. 7, pp. 833-844, 1999.

[22] S. Zheng, "Global boundedness of solutions to a reactiondiffusion system," Mathematical Methods in the Applied Sciences, vol. 22, no. 1, pp. 43-54, 1999.

[23] S. Zheng, "Global existence and global non-existence of solutions to a reaction-diffusion system," Nonlinear Analysis: Theory, Methods \& Applications, vol. 39, no. 3, pp. 327-340, 2000.

[24] W. A. Day, "A decreasing property of solutions of parabolic equations with applications to thermoelasticity," Quarterly of Applied Mathematics, vol. 40, no. 4, pp. 468-475, 1983.

[25] W. A. Day, Heat Conduction within Linear Thermoelasticity, vol. 30 of Springer Tracts in Natural Philosophy, Springer, New York, NY, USA, 1985. 
[26] A. Friedman, "Monotonic decay of solutions of parabolic equations with nonlocal boundary conditions," Quarterly of Applied Mathematics, vol. 44, no. 3, pp. 401-407, 1986.

[27] C. V. Pao, "Asymptotic behavior of solutions of reactiondiffusion equations with nonlocal boundary conditions," Journal of Computational and Applied Mathematics, vol. 88, no. 1, pp. 225-238, 1998.

[28] C. V. Pao, "Numerical solutions of reaction-diffusion equations with nonlocal boundary conditions," Journal of Computational and Applied Mathematics, vol. 136, no. 1-2, pp. 227-243, 2001.

[29] S. Seo, "Blowup of solutions to heat equations with nonlocal boundary conditions," Kobe Journal of Mathematics, vol. 13, no. 2, pp. 123-132, 1996.

[30] L.-H. Kong and M.-X. Wang, "Global existence and blow-up of solutions to a parabolic system with nonlocal sources and boundaries," Science in China A, vol. 50, no. 9, pp. 1251-1266, 2007.

[31] S. Zheng and L. Kong, "Roles of weight functions in a nonlinear nonlocal parabolic system," Nonlinear Analysis: Theory, Methods \& Applications, vol. 68, no. 8, pp. 2406-2416, 2008.

[32] K. Deng, "Comparison principle for some nonlocal problems," Quarterly of Applied Mathematics, vol. 50, no. 3, pp. 517-522, 1992.

[33] A. Friedman and B. McLeod, "Blow-up of positive solutions of semilinear heat equations," Indiana University Mathematics Journal, vol. 34, no. 2, pp. 425-447, 1985. 


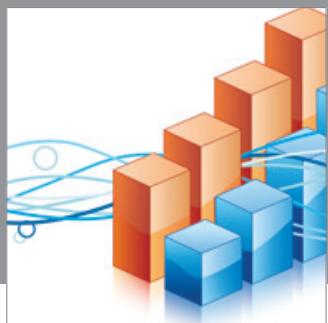

Advances in

Operations Research

mansans

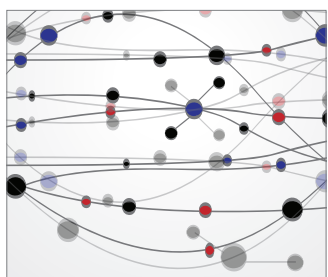

The Scientific World Journal
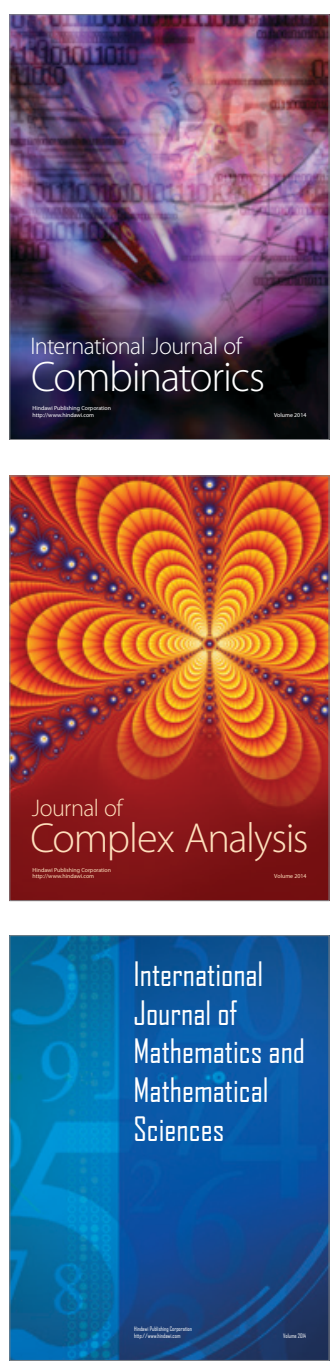
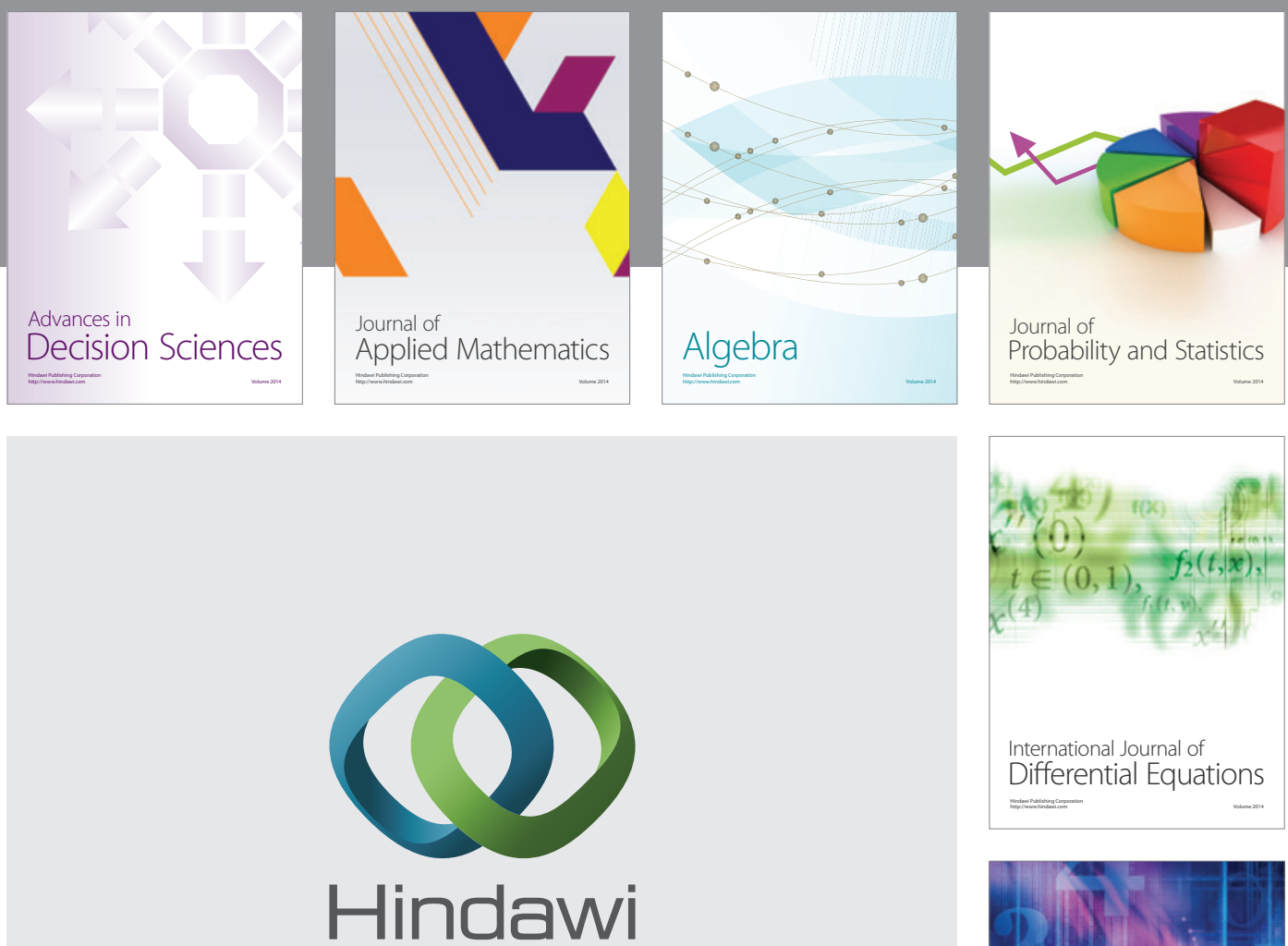

Submit your manuscripts at http://www.hindawi.com
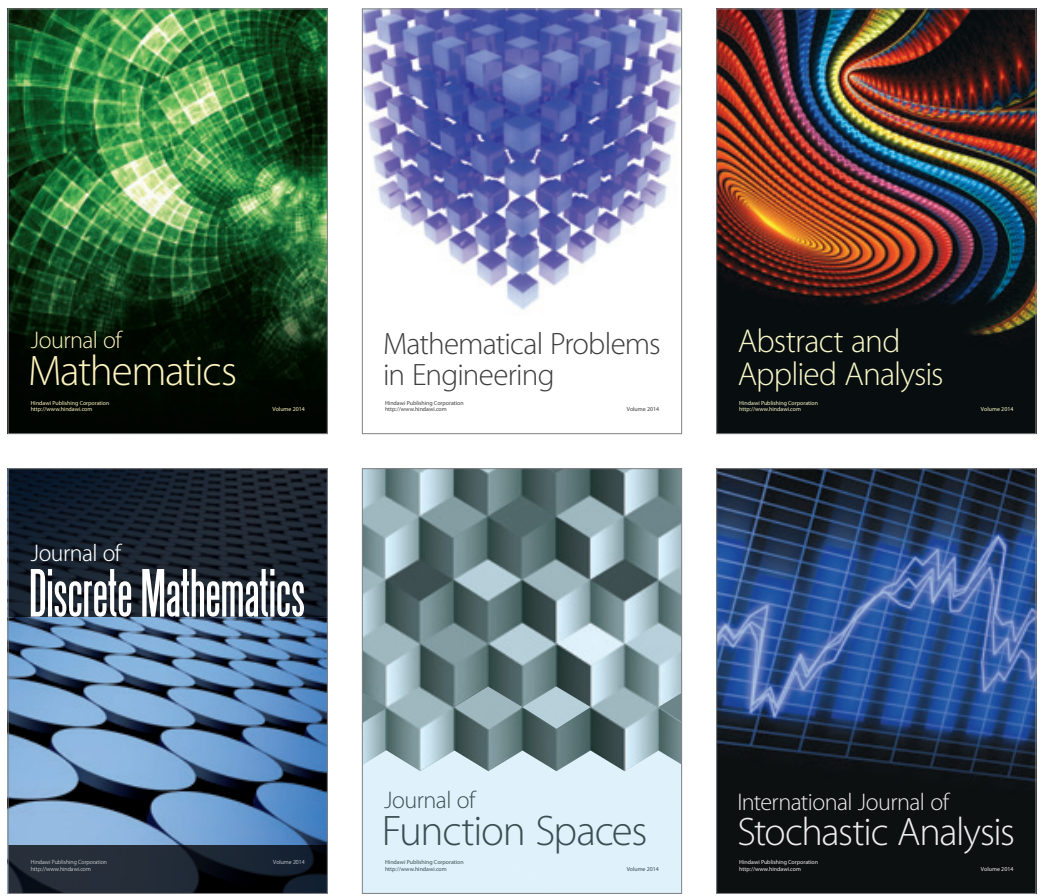

Journal of

Function Spaces

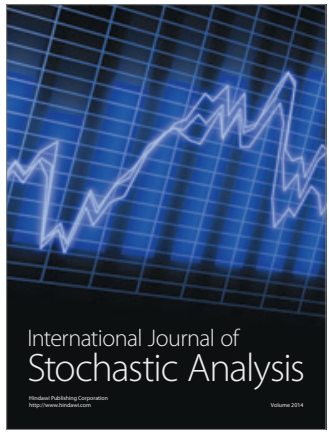

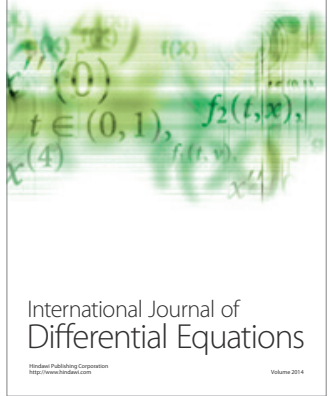
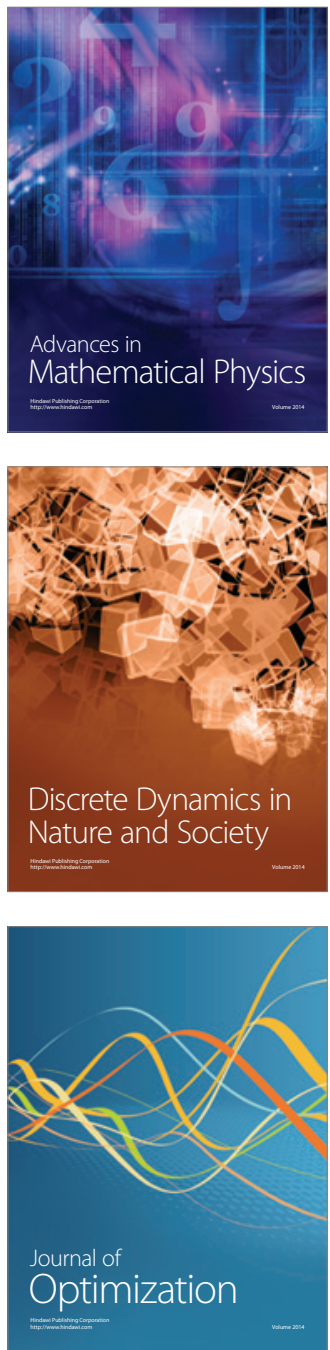